\title{
Multireference Perturbation Theory with Optimized Partitioning. II. Applications to Molecular Systems
}

\author{
HENRYK A. WITEK, HARUYUKI NAKANO, KIMIHIKO HIRAO \\ Department of Applied Chemistry, School of Engineering, The University of Tokyo, \\ Tokyo 113-8656, Japan
}

Received 27 January 2003; Accepted 10 April 2003

\begin{abstract}
The second-order multireference perturbation theory using an optimized partitioning, denoted as MROPT(2), is applied to calculations of various molecular properties-excitation energies, spectroscopic parameters, and potential energy curves - for five molecules: ethylene, butadiene, benzene, $\mathrm{N}_{2}$, and $\mathrm{O}_{2}$. The calculated results are compared with those obtained with second- and third-order multireference perturbation theory using the traditional partitioning techniques. We also give results from computations using the multireference configuration interaction (MRCI) method. The presented results show very close resemblance between the new method and MRCI with renormalized Davidson correction. The accuracy of the new method is good and is comparable to that of second-order multireference perturbation theory using Møller-Plesset partitioning.
\end{abstract}

(C) 2003 Wiley Periodicals, Inc. J Comput Chem 24: 1390-1400, 2003

Key words: multireference perturbation theory; optimized partitioning; excitation energy; potential energy surface (PES)

\section{Introduction}

One of the most popular strategies for solving the energy eigenvalue problem in quantum chemistry is perturbation theory (PT). In PT, the Hamiltonian, $\hat{H}$, is divided into two operators: $\hat{H}_{0}$, called a zeroth-order Hamiltonian, and $\hat{V}$, which is called a perturbation. Subsequently, the eigenvalue problem corresponding to the zeroth-order Hamiltonian is solved exactly-usually using a variational procedure-yielding a complete set of zeroth-order wave functions $\left\{\Psi_{j}^{(0)}\right\}$ and zeroth-order energies $\left\{E_{j}^{(0)}\right\}$. These quantities, together with the operator $\hat{V}$, are used then to obtain the exact eigenfunctions and eigenvalues of the original eigenvalue problem in a perturbative manner.

The division of $\hat{H}$ into $\hat{H}_{0}$ and $\hat{V}$-called a partitioning of the Hamiltonian - is the most crucial point of every perturbative treatment. In many cases, the partitioning is implied by the physics of the problem. However, this is not the case when computing correlation energy in atomic and molecular systems. As is well known, it makes no difference which partitioning is used when the perturbation series is considered up to the infinite order, providing, of course, the convergent character of the perturbational expansions. However, in practical applications, no infinite perturbation series is used. Usually, the series is terminated at some low order, yielding an appropriate $n$ th-order ansatz for the wave function. The higher-order terms, $\Psi_{\alpha}^{(m)}$, where $m>n$, are assumed to be zero and are neglected. This is a good approximation for fast-converg- ing series. Unfortunately, neither the Møller-Plesset partitioning ${ }^{1}$ nor the Epstein-Nesbet partitioning ${ }^{2,3}$ - the two most popular ways of defining $\hat{H}_{\mathrm{O}}$ in quantum chemistry-produces fast converging series. Therefore, neglecting the higher-order terms may result in introducing some errors. There is no systematic way of estimating these errors, because usually the higher-order wave functions are not computed. In contrast to variational techniques, where the calculated energy constitutes a natural upper bound to the exact energy, there are usually no such bounds for low-order PT. (The situation is similar for the coupled cluster methods.) This lack of possibility of determining the accuracy of the PT results is one of the most serious drawbacks of perturbation theory.

One of the possible solutions to this problem can be using an optimized partitioning that ensures fast convergence of the PT series. A few propositions of such methods have been made. $\mathrm{Amos}^{4}$ used a single variational parameter that was adjusted to make the third-order energy vanish, $E^{(3)}=0$. Multiple variational parameters-which can be interpreted as the zeroth-order energies of the states from the first-order interacting space (FOCI) - were used by Szabados and Surján., ${ }^{5,6}$ These parameters were determined by minimizing the energy in the Rayleigh quotient taken with the first-order perturbational ansatz for the wave function.

Correspondence to: H. A. Witek; e-mail: cedziu@qcl.t.u-tokyo.ac.jp Contract/grant sponsor: Ministry of Education, Culture, Sports, Science, and Technology, Japan 
Finley optimized the zeroth-order energies of the FOCI's states using a concept of maximum radius of convergence, ${ }^{7}$ derived from a two-state model. Another approach of Finley and coworkers ${ }^{8,9}$ used a small subspace of FOCI, which contains a set of the most important configuration state functions, to optimize the zerothorder energies of states belonging to this subspace. The optimization was performed by minimizing some energy functional that comprised the differences between the exact energy and third- and fourth-order perturbational energy; all quantities were determined within the chosen subspace. All these methods-except for the maximum radius of convergence partitioning, for which no numerical tests have yet been performed-showed much better convergence characteristics than the traditional partitionings. Unfortunately, the proposed methods do not allow for optimization of the zeroth-order energies of states outside of FOCI.

In the preceding article,${ }^{10}$ we have proposed a family of optimized zeroth-order Hamiltonians that allow for partial control of the errors arising from truncation of perturbational series. Using this new partitioning enables optimizing of the zeroth-order energies of all states appearing in perturbational expansion. Some theoretical and numerical aspects of one of the resulting methods-abreviated as MROPT(2) - have been analyzed and discussed. In the present article, we apply this second-order multireference PT with the optimized partitioning to calculations on some molecular properties of five molecules: ethylene, butadiene, benzene, and molecular nitrogen and oxygen. The calculated results are preceded by a brief exposition of the theory of optimized partitioning. For detailed derivations, see the preceding article. ${ }^{10}$

\section{Theory}

Let $|\alpha\rangle$ be a multideterminantal wave function corresponding to the ground or some excited state $\alpha$ of a given molecular system. The wave function $|\alpha\rangle$ is obtained by diagonalizing the matrix of the Hamiltonian operator $\hat{H}$ within a chosen set of the most important configurations state functions (CSFs), called a reference space. [The most popular choice of a reference space is a complete active space (CAS).] Such a multideterminantal wave function $|\alpha\rangle$ accounts for nondynamical correlation effects and describes well the near-degeneracy effects. In order to obtain an accurate estimation of the energy of the state $\alpha$, we treat dynamical correlation by means of multireference PT. The matrix representations of all operators are given in a space spanned by the multideterminantal states $|\alpha\rangle,\left|k_{1}\right\rangle,\left|k_{1}\right\rangle, \ldots$, obtained by diagonalizing $\hat{H}$ within the reference space, and all nonredundant CSFs $\left|q_{1}\right\rangle,\left|q_{2}\right\rangle, \ldots$ obtained by applying single, double, triple, and higher excitation to the reference space's CSFs. The set of all singly- and doublyexcited CSFs constitutes the FOCI.

We define the zeroth-order Hamiltonian $\hat{H}_{0}$ as a diagonal matrix operator:

$$
\left(H_{0}\right)_{i j}=\delta_{i j} E_{i}^{(0)}
$$

where the zeroth-order energies $E_{i}^{(0)}$ are defined by

$$
E_{i}^{(0)}= \begin{cases}H_{\alpha \alpha} & \text { if } i=\alpha ; \\ H_{i i}+\Delta_{i} & \text { if } i=k_{s}, q_{s}\end{cases}
$$

The matrix of perturbation operator $\hat{V}$ is given by

$$
V_{i j}=H_{i j}-\left(H_{0}\right)_{i j}= \begin{cases}H_{i j} & \text { if } i \neq j \\ 0 & \text { if } i=j=\alpha \\ -\Delta_{i} & \text { if } i=j \neq \alpha\end{cases}
$$

The state-dependent parameters $\Delta_{i}$ are at our disposal; we are going to adjust them in such a manner that the first neglected term, $\Psi_{\alpha}^{(n+1)}$, in the $n$ th-order perturbational ansatz for wave function

$$
\Psi_{\alpha}^{[n]}=|\alpha\rangle+\Psi_{\alpha}^{(1)}+\cdots+\Psi_{\alpha}^{(n)}
$$

is identically equal to zero,

$$
\Psi_{\alpha}^{(n+1)}=0
$$

This condition allows for a partial control of errors associated with truncating the perturbational expansion of the wave function series at the $n$ th-order. Eq. (5) defines the set of state-dependent parameters $\Delta_{i}$ in an implicit way. It is difficult to give a compact, explicit equation defining $\Delta_{i}$ in a general case. The explicit set of linear equations defining $\Delta_{i}$ for the second-order multireference PT with optimized partitioning is given by

$$
\Psi_{\alpha}^{(2)}=0 \Leftrightarrow \forall j: \sum_{s \neq \alpha} H_{\alpha s}\left(H_{s j}-\delta_{s j} E_{\alpha}^{(0)}\right) \Lambda_{s}=H_{\alpha j}
$$

where

$$
\Lambda_{i}=\frac{1}{H_{i i}+\Delta_{i}-E_{\alpha}^{(0)}}
$$

Explicit equations for the third- and fourth-order PT were given in our previous article. ${ }^{10}$ By saying " $n$ th-order PT" we mean-as usual in quantum chemistry - that the highest retained term in energy series is $E_{\alpha}^{(n)}$. To calculate this term, it is sufficient to terminate wave function expansion at the $(n-1)$ th-order, that is, the highest retained term is $\Psi_{\alpha}^{(n-1)}$. Note that eq. (6) is formally identical to the working equations of the linearized multireference coupled cluster (CC) method ${ }^{11,12}$ and the optimized partitioning of Szabados and Surján. ${ }^{5,6}$

Second-order multireference Rayleigh-Schrödinger perturbation theory with optimized partitioning is uniquely defined by eqs. (1), (3), and (6), provided that the reference space and one-electron orbitals have been determined. The first-order wave function is given by

$$
\Psi_{\alpha}^{(1)}=-\sum_{q \in \mathrm{FOCI}} H_{\alpha q} \Lambda_{q}|q\rangle
$$

Energy of the state $\alpha$ through the second-order of PT is given by

$$
E_{\alpha}=E_{\alpha}^{(0)}-\sum_{q \in \mathrm{FOCI}} H_{\alpha q}^{2} \Lambda_{q}
$$


Table 1. Excitation Energies (eV) for Two States of Ethylene and Four States of Butadiene Calculated Using the MROPT(2) Method.

cc-pVDZ basis set

\begin{tabular}{|c|c|c|c|c|c|c|c|}
\hline \multirow[b]{2}{*}{ Method } & \multicolumn{2}{|c|}{ Ethylene } & \multicolumn{4}{|c|}{ Butadiene } & \multirow[b]{2}{*}{ Error } \\
\hline & $1{ }^{1} B_{1 u}$ & $1{ }^{3} B_{1 u}$ & $2{ }^{1} A_{g}$ & $1{ }^{1} B_{u}$ & $1{ }^{3} B_{u}$ & $1{ }^{3} A_{g}$ & \\
\hline CASSCF & 10.08 & 4.34 & 6.79 & 8.50 & 3.42 & 5.14 & 0.80 \\
\hline MRCI & 9.11 & 4.53 & 6.83 & 7.41 & 3.46 & 5.27 & 0.51 \\
\hline $\mathrm{MRCI}+\mathrm{Q}$ & 8.76 & 4.56 & 6.79 & 6.91 & 3.47 & 5.30 & 0.38 \\
\hline $\operatorname{MREN}(2)$ & 7.85 & 4.61 & 7.56 & 5.40 & 3.40 & 5.57 & 0.48 \\
\hline MREN(3) & 9.30 & 4.44 & 6.74 & 7.44 & 3.43 & 5.14 & 0.51 \\
\hline MRMP(2) & 8.61 & 4.52 & 6.57 & 6.41 & 3.27 & 5.08 & 0.23 \\
\hline MRMP(3) & 8.87 & 4.47 & 8.02 & 6.92 & 3.40 & 5.15 & 0.55 \\
\hline MROPT(2) & 8.55 & 4.55 & 6.83 & 6.52 & 3.47 & 5.30 & 0.28 \\
\hline
\end{tabular}

cc-pVTZ basis set

\begin{tabular}{|c|c|c|c|c|c|c|c|}
\hline \multirow[b]{2}{*}{ Method } & \multicolumn{2}{|c|}{ Ethylene } & \multicolumn{4}{|c|}{ Butadiene } & \multirow[b]{2}{*}{ Error } \\
\hline & $1{ }^{1} B_{1 u}$ & $1{ }^{3} B_{1 u}$ & $2{ }^{1} A_{g}$ & $1{ }^{1} B_{u}$ & $1{ }^{3} B_{u}$ & $1{ }^{3} A_{g}$ & \\
\hline CASSCF & 9.57 & 4.31 & 6.75 & 8.31 & 3.41 & 5.12 & 0.69 \\
\hline MRCI & 8.77 & 4.50 & 6.77 & 7.24 & 3.45 & 5.22 & 0.41 \\
\hline $\mathrm{MRCI}+\mathrm{Q}$ & 8.42 & 4.53 & 6.72 & 6.71 & 3.46 & 5.25 & 0.27 \\
\hline MREN(2) & 7.69 & 4.60 & 7.49 & 5.01 & 3.38 & 5.52 & 0.56 \\
\hline MREN(3) & 8.92 & 4.40 & 6.66 & 7.28 & 3.41 & 5.08 & 0.40 \\
\hline MRMP(2) & 8.29 & 4.45 & 6.40 & 6.10 & 3.20 & 4.95 & 0.15 \\
\hline MRMP(3) & 8.58 & 4.44 & 8.13 & 6.71 & 3.39 & 5.10 & 0.48 \\
\hline MROPT(2) & 8.04 & 4.53 & 6.75 & 6.18 & 3.46 & 5.25 & 0.15 \\
\hline Exp. & $\approx 8.0^{\mathrm{a}}$ & $4.36^{\mathrm{b}}$ & $\ldots$ & $6.25^{\mathrm{c}}$ & $3.22^{\mathrm{c}}$ & $4.91^{\mathrm{c}}$ & \\
\hline
\end{tabular}

The results obtained using other methods are given for comparison. Error is computed as an average absolute deviation from the experimental results. For the $2{ }^{1} A_{g}$ state of butadiene, for which no experimental data are available, the corresponding $\mathrm{MRCI}+\mathrm{Q}$ energies are used as a reference.

${ }^{a}$ Estimated vertical excitation energy from earlier theoretical work (refs. 32-35).

${ }^{\mathrm{b}}$ Refs. 36, 37.

${ }^{\mathrm{c}}$ Ref. 38.

The second-order wave function $\Psi_{\alpha}^{(2)}$ is identically equal to zero [see eq. (6)]. As a consequence, the third-order energy also vanishes. The first nonvanishing correction to energy is $E_{\alpha}^{(4)}$. Vanishing of $\Psi_{\alpha}^{(2)}$ has some deeper consequences: Surján and Szabados showed $^{6}$ that most components of the fifth-order energy also vanish. Recently, this optimized partitioning has been applied within a framework of multireference PT to correct the quality of energies and wave functions obtained using limited CC methods, ${ }^{13}$ showing significantly better performance than the MP and Dysonlike ${ }^{14}$ partitionings.

For further use, the second-order multireference RayleighSchrödinger PT with optimized partitioning is referred to as the MROPT(2) method.

\section{Computational Details}

The planar geometries of ethylene and butadiene are taken from experiment. For ethylene, ${ }^{15}$ the bond lengths are $r_{\mathrm{CC}}=1.339 \AA$ and $r_{\mathrm{CH}}=1.086 \AA$, and the $\angle \mathrm{CCH}$ angle is $117.6^{\circ}$. For butadiene, ${ }^{16}$ the bond lengths are $r_{\mathrm{C}_{1} \mathrm{C}_{2}}=1.467 \AA, r_{\mathrm{C}_{2} \mathrm{C}_{3}}=1.343 \AA$, and $r_{\mathrm{CH}}=1.094 \AA$, and the angles are $\angle \mathrm{CCC}=122.8^{\circ}$ and $\angle \mathrm{CCH}=119.5^{\circ}$. For benzene, we use also a planar hexagonal geometry with following bond lengths: $r_{\mathrm{CC}}=1.395 \AA$ and $r_{\mathrm{CH}}=$ $1.084 \AA$, which are very close to the experimental data. ${ }^{15}$ For calculations on spectroscopic parameters of $\mathrm{O}_{2}$ and $\mathrm{N}_{2}$, we usually use seven different geometries in the range $\left[r_{e}-0.1 \AA, r_{e}+0.1\right.$ $\AA$ ], where $r_{e}$ is the equilibrium geometry of a given state. The geometries used for the calculations on the internal rotation potential energy curve in ethylene are optimized on the B3LYP/6$31++\mathrm{G}(2 d, 2 p)$ level with the dihedral angle kept frozen. The excitation energy calculations for ethylene and butadiene are performed with the cc-pVDZ (correlation-consistent polarized valence double zeta) and cc-pVTZ (correlation-consistent polarized valence triple zeta) basis sets of Dunning. ${ }^{17}$ For calculations on internal rotation potential energy curve in ethylene we use ANO (atomic natural orbitals) type orbitals, ${ }^{18}$ with a $(10 s 6 p 3 d) /$ $[7 s 6 p 3 d]$ contraction scheme for $\mathrm{C}$ and $(7 s 3 p) /[6 s 3 p]$ for $\mathrm{H}$. 
Table 2. Excitation Energies (eV) for Eight States of Benzene Calculated Using the MROPT(2) Method.

\begin{tabular}{lllllllll}
\hline Method & ${ }^{1} E_{2 g}$ & ${ }^{1} B_{1 u}$ & ${ }^{1} B_{2 u}$ & ${ }^{1} E_{1 u}$ & ${ }^{3} E_{2 g}$ & ${ }^{3} B_{1 u}$ & ${ }^{3} B_{2 u}$ & ${ }^{3} E_{1 u}$ \\
\hline CASSCF & 8.17 & 7.85 & 4.97 & 9.30 & 7.20 & 3.87 & 7.09 & 5.00 \\
MRCI & 8.27 & 7.03 & 5.09 & 8.16 & 7.42 & 4.10 & 6.32 & 4.96 \\
MRCI+Q & 8.24 & 6.63 & 5.10 & 7.51 & 7.47 & 4.18 & 5.95 & 4.90 \\
MREN(2) & 9.54 & 5.52 & 6.06 & 5.40 & 8.39 & 4.60 & 4.26 & 5.01 \\
MREN(3) & 8.16 & 6.60 & 5.02 & 8.13 & 7.31 & 4.06 & 6.13 & 4.90 \\
MRMP(2) & 7.71 & 6.07 & 4.61 & 6.58 & 6.99 & 3.93 & 5.41 & 4.43 \\
MRMP(3) & 9.48 & 6.37 & 5.32 & 9.48 & 8.42 & 4.12 & 6.28 & 5.11 \\
MROPT(2) & 8.24 & 6.22 & 5.09 & $\ldots$ & 7.47 & 4.19 & 5.60 & 0.41 \\
Exp. & $7.80^{\mathrm{a}}$ & $6.20^{\mathrm{b}}$ & $4.90^{\mathrm{b}}$ & $6.94^{\mathrm{b}}$ & $6.83^{\mathrm{c}}$ & $3.95^{\mathrm{d}}$ & $5.60^{\mathrm{d}}$ & 4.90 \\
\hline
\end{tabular}

The results computed using other methods are given for comparison. Error is computed as an average absolute deviation from the experimental results.

${ }^{\text {aRef. }} 39$.

${ }^{\mathrm{b}}$ Ref. 40 .

${ }^{\mathrm{c}}$ Ref. 41.

${ }^{\mathrm{d} R e f .} 42$.

Similarly, in the calculations of excitation energies of benzene, we use an ANO-type ${ }^{19}(14 s 9 p 4 d) /[4 s 3 p 2 d]$ contraction scheme for carbon and $(8 s 4 p) /[3 s 2 p]$ for hydrogen. The basis set used for $\mathrm{N}_{2}$ is an ANO-type ${ }^{18}(10 s 6 p 3 d) /[4 s 3 p 2 d]$ contraction scheme and the basis set used for $\mathrm{O}_{2}$ is an ANO-type ${ }^{19}(14 s 9 p 4 d 3 f) /$ $[4 s 3 p 2 d 1 f]$ contraction scheme.

All calculations use CASSCF reference wave functions. The active space in the calculations of excitation energies and rotational barrier for ethylene is $(2 e, 2 o)$; the orbitals are HOMO $\pi$ and LUMO $\pi^{*}$. The active space for butadiene is $(4 e, 4 o)$; the orbitals are HOMO $\pi_{1}, \pi_{2}$ and LUMO $\pi_{1}^{*}, \pi_{2}^{*}$. For benzene, CAS consists of six electrons and six orbitals, $a_{2 u}, e_{1 g}, e_{2 u}$, and $b_{2 g}$, where the symmetry labels of orbitals are given in $D_{6 h}$ symmetry. In perturbational calculations, the $1 s$ orbital of carbon atoms is kept frozen for all computed hydrocarbons. Moreover, for benzene, we freeze the 51 highest virtual orbitals, because otherwise the dimension of the first-order interacting space is too large to perform the MRCI and MRPT calculations. The number of frozen virtuals for each $D_{2 h}$ irreducible representation is: 11 of $A_{g}$ and $B_{1 g}$, one of $B_{2 g}$, three of $B_{3 g}$, two of $A_{u}$ and $B_{1 u}, 13$ of $B_{2 u}$, and eight of $B_{3 u}$. In calculations for $\mathrm{N}_{2}$ and $\mathrm{O}_{2}$, we are using the $(6 e$, $8 o)$ and $(12 e, 8 o)$ active spaces, respectively. The orbitals are $\sigma_{2 p_{z}}, \pi_{2 p_{x}}, \pi_{2 p_{y}}, \pi_{2 p_{x}}^{*}, \pi_{2 p_{y}}^{*}, \sigma_{2 p_{z}}^{*}, \sigma_{3 s}$, and $\sigma_{3 s}^{*}$ for $\mathrm{N}_{2}$, and $\sigma_{2 s}$, $\sigma_{2 s}^{*}, \sigma_{2 p_{z}}, \pi_{2 p_{x}}, \pi_{2 p_{y}}, \pi_{2 p_{x}}^{*}, \pi_{2 p_{y}}^{*}$, and $\sigma_{2 p_{z}}^{*}$ for $\mathrm{O}_{2}$. In perturbational calculations, the $1 s$ orbital of nitrogen atoms are kept frozen. For all molecules, a set of state-specific CASSCF orbitals is used for every calculation. The orbitals for high-symmetry states, the $\Pi$ and $\Delta$ states of $\mathrm{N}_{2}$ and $\mathrm{O}_{2}$ and the $E_{2 g}$ and $E_{1 u}$ states of benzene, are obtained by including both $D_{2 h}$ components of these states in the CASSCF optimization.

All CASSCF, MRCI, and perturbational calculations are performed using COLUMBUS, a collection of programs for highlevel ab initio molecular electronic structure calculations. ${ }^{20-23}$ The perturbative methods use a modified multireference configuration interaction (MRCI) code of COLUMBUS. The modifications concern mostly the way of using the graphical unitary group approach (GUGA)-based matrix-vector multiplication routine and some mi- nor changes in the existing Fock-matrix calculation routine. A new PT-driver routine has been added, along with routines that allow solving large sets of linear equations using iterative techniques.

\section{Results}

MROPT(2) is applied for calculating various molecular properties of ground and excited states for a set of small and medium size molecules. We compute valence excitation energies of ethylene, butadiene, and benzene, spectroscopic parameters of six states of $\mathrm{O}_{2}$ and eight states of $\mathrm{N}_{2}$, and the height of the internal rotation barrier of ethylene. We give also a comparison of the MROPT(2) results to those obtained with other methods, that is, MRCI method, MRCI with renormalized Davidson correction ${ }^{24}$ (MRCI+Q), second- and third-order multireference PT using Epstein-Nesbet partitioning [denoted as $\operatorname{MREN}(2)$ and $\operatorname{MREN}(3)$, respectively], and second- and third-order multireference PT using Møller-Plesset partitioning [denoted as MRMP(2) and MRMP(3), respectively].

\section{Valence Excitation Energies of Ethylene, Butadiene, and Benzene}

Excitation energies for two states of ethylene, $1{ }^{1} B_{1 u}$ and $1{ }^{3} B_{1 u}$, and for four states of butadiene, $2{ }^{1} A_{g}, 1{ }^{1} B_{u}, 1{ }^{3} B_{u}$, and $1{ }^{3} A_{g}$ are given in Table 1. Presented results depend rather weakly on the quality of the basis set used for calculations, except for the $1{ }^{1} B_{1}$ state of ethylene and the $1{ }^{1} B_{u}$ state of butadiene. This is rather easy to explain if we remember that these two states have an ionic-like $\left[\mathrm{C}^{+} \mathrm{C}^{-}\right]+\left[\mathrm{C}^{-} \mathrm{C}^{+}\right]$character in a valence bond $(\mathrm{VB})$ description. ${ }^{25-28}$ Improving the quality of the basis set allows for better description of the ionic-like $\mathrm{C}^{-}$centers and lowers the energy of the state by a large amount. The other states-which are predominantly composed of the covalent Kekule- and Dewar-like structures-do not show this effect. The MROPT(2) excitation energies for the covalent-like states are almost identical to the 
Table 3. Spectroscopic Parameters for Six States of $\mathrm{O}_{2}$ Determined Using the MROPT(2) Method.

\begin{tabular}{lcccccc}
\hline \multicolumn{7}{c}{ Equilibrium distance $r_{e}$} \\
\hline Method & $b^{1} \Sigma_{g}^{+}$ & $c^{1} \Sigma_{u}^{-}$ & $a^{1} \Delta_{g}$ & $A^{\prime}{ }^{3} \Delta_{u}$ & $X^{3} \Sigma_{g}^{-}$ & $A^{3} \Sigma_{u}^{+}$ \\
\hline CASSCF & 1.244 & 1.592 & 1.231 & 1.576 & 1.218 & 1.587 \\
MRCI & 1.235 & 1.532 & 1.223 & 1.527 & 1.213 & 1.534 \\
MRCI+Q & 1.238 & 1.533 & 1.225 & 1.530 & 1.217 & 1.537 \\
MREN(2) & 1.236 & 1.547 & 1.225 & 1.542 & 1.217 & 0.041 \\
MREN(3) & 1.234 & 1.520 & 1.220 & 1.516 & 1.210 & 1.550 \\
MRMP(2) & 1.217 & 1.476 & 1.208 & 1.474 & 1.202 & 0.013 \\
MRMP(3) & 1.241 & 1.536 & 1.229 & 1.533 & 1.218 & 1.483 \\
MROPT(2) & 1.239 & 1.528 & 1.226 & 1.529 & 1.218 & 1.544 \\
Exp. ${ }^{a}$ & 1.227 & 1.514 & 1.216 & 1.513 & 1.208 & 0.004 \\
\hline
\end{tabular}

Harmonic vibrational frequency $\omega_{e}$

\begin{tabular}{lccccrr}
\hline Method & $b^{1} \Sigma_{g}^{+}$ & $c{ }^{1} \Sigma_{u}^{-}$ & $a^{1} \Delta_{g}$ & $A^{\prime}{ }^{3} \Delta_{u}$ & $X{ }^{3} \Sigma_{g}^{-}$ & $A{ }^{3} \Sigma_{u}^{+}$ \\
\hline CASSCF & 1396 & 554 & 1462 & 601 & 1564 & 574 \\
MRCI & 1429 & 750 & 1517 & 773 & 1591 & 761 \\
MRCI+Q & 1417 & 781 & 1505 & 800 & 1577 & 786 \\
MREN(2) & 1422 & 738 & 1504 & 755 & 1578 & 740 \\
MREN(3) & 1429 & 794 & 1526 & 818 & 1610 & 804 \\
MRMP(2) & 1531 & 964 & 1619 & 983 & 1674 & 968 \\
MRMP(3) & 1397 & 733 & 1475 & 747 & 1556 & 720 \\
MROPT(2) & 1412 & 767 & 1500 & 787 & 1567 & 774 \\
Exp. ${ }^{a}$ & 1433 & 797 & 1509 & 815 & 1580 & 504 \\
\hline
\end{tabular}

Rotational constant $B_{e}$

\begin{tabular}{|c|c|c|c|c|c|c|c|}
\hline Method & $b^{1} \Sigma_{g}^{+}$ & $c^{1} \Sigma_{u}^{-}$ & $a^{1} \Delta_{g}$ & $A^{\prime}{ }^{3} \Delta_{u}$ & $X^{3} \Sigma_{g}^{-}$ & $A^{3} \Sigma_{u}^{+}$ & Error \\
\hline CASSCF & 1.36 & 0.83 & 1.39 & 0.85 & 1.42 & 0.84 & 0.05 \\
\hline MRCI & 1.38 & 0.90 & 1.41 & 0.90 & 1.43 & 0.90 & 0.01 \\
\hline $\mathrm{MRCI}+\mathrm{Q}$ & 1.37 & 0.90 & 1.40 & 0.90 & 1.42 & 0.89 & 0.02 \\
\hline MREN(2) & 1.38 & 0.88 & 1.40 & 0.89 & 1.42 & 0.88 & 0.02 \\
\hline MREN(3) & 1.38 & 0.91 & 1.41 & 0.92 & 1.44 & 0.91 & 0.00 \\
\hline MRMP(2) & 1.42 & 0.97 & 1.44 & 0.97 & 1.46 & 0.96 & 0.03 \\
\hline MRMP(3) & 1.37 & 0.89 & 1.40 & 0.90 & 1.42 & 0.88 & 0.02 \\
\hline MROPT(2) & 1.37 & 0.90 & 1.40 & 0.90 & 1.42 & 0.89 & 0.02 \\
\hline Exp. $^{a}$ & 1.40 & 0.92 & 1.43 & 0.92 & 1.44 & 0.91 & \\
\hline
\end{tabular}

\begin{tabular}{|c|c|c|c|c|c|c|c|}
\hline \multicolumn{8}{|c|}{ Adiabatic excitation energy } \\
\hline Method & $b^{1} \Sigma_{g}^{+}$ & $c^{1} \Sigma_{u}^{-}$ & $a^{1} \Delta_{g}$ & $A^{\prime}{ }^{3} \Delta_{u}$ & $X^{3} \Sigma_{g}^{-}$ & $A^{3} \Sigma_{u}^{+}$ & Error \\
\hline CASSCF & 1.46 & 3.72 & 0.95 & 3.95 & 0.00 & 4.00 & 0.26 \\
\hline MRCI & 1.63 & 4.01 & 1.00 & 4.23 & 0.00 & 4.29 & 0.06 \\
\hline $\mathrm{MRCI}+\mathrm{Q}$ & 1.68 & 4.00 & 1.03 & 4.23 & 0.00 & 4.29 & 0.07 \\
\hline $\operatorname{MREN}(2)$ & 1.68 & 4.05 & 1.02 & 4.28 & 0.00 & 4.35 & 0.04 \\
\hline MREN(3) & 1.62 & 3.86 & 0.96 & 4.07 & 0.00 & 4.15 & 0.15 \\
\hline $\operatorname{MRMP}(2)$ & 1.83 & 4.68 & 1.10 & 4.69 & 0.00 & 4.55 & 0.28 \\
\hline MRMP(3) & 1.56 & 3.81 & 0.95 & 4.02 & 0.00 & 4.14 & 0.18 \\
\hline MROPT(2) & 1.65 & 3.92 & 1.01 & 4.18 & 0.00 & 4.27 & 0.09 \\
\hline Exp. $^{\mathrm{a}}$ & 1.64 & 4.10 & 0.98 & 4.31 & 0.00 & 4.39 & \\
\hline
\end{tabular}


Table 3. (Continued)

\begin{tabular}{|c|c|c|c|c|c|c|c|}
\hline \multicolumn{8}{|c|}{ Vertical excitation energy } \\
\hline Method & $b^{1} \Sigma_{g}^{+}$ & $c^{1} \Sigma_{u}^{-}$ & $a^{1} \Delta_{g}$ & $A^{\prime}{ }^{3} \Delta_{u}$ & $X^{3} \Sigma_{g}^{-}$ & $A^{3} \Sigma_{u}^{+}$ & Error \\
\hline CASSCF & 1.50 & 5.86 & 0.97 & 6.09 & 0.00 & 6.21 & 0.16 \\
\hline MRCI & 1.65 & 6.01 & 1.00 & 6.24 & 0.00 & 6.36 & 0.04 \\
\hline MREN(2) & 1.70 & 6.13 & 1.03 & 6.37 & 0.00 & 6.51 & 0.05 \\
\hline MREN(3) & 1.64 & 5.82 & 0.97 & 6.03 & 0.00 & 6.17 & 0.16 \\
\hline MRMP(2) & 1.83 & 6.43 & 1.10 & 6.47 & 0.00 & 6.42 & 0.15 \\
\hline $\operatorname{MRMP}(3)$ & 1.59 & 5.82 & 0.97 & 6.04 & 0.00 & 6.22 & 0.16 \\
\hline MROPT(2) & 1.68 & 5.70 & 1.02 & 6.21 & 0.00 & 6.38 & 0.09 \\
\hline $\mathrm{MRCI}+\mathrm{Q}$ & 1.70 & 6.03 & 1.03 & 6.29 & 0.00 & 6.41 & \\
\hline
\end{tabular}

Results obtained using other methods are given for comparison. Distances are given in $\AA$, vibrational frequencies and rotational constants in $\mathrm{cm}^{-1}$, and excitation energies in $\mathrm{eV}$. Error is computed as an average absolute deviation from the experimental results. For vertical excitation energies, no experimental data are available and error is calculated with respect to the $\mathrm{MRCI}+\mathrm{Q}$ parameters.

${ }^{a}$ Experimental data for the $b^{1} \Sigma_{g}^{+}, a^{1} \Delta_{g}$, and $X^{3} \Sigma_{g}^{-}$states are taken from ref. 43. Experimental data for the $c^{1} \Sigma_{u}^{-}$, $A^{\prime}{ }^{3} \Delta_{u}$, and $A^{3} \Sigma_{u}^{+}$states are taken from ref. 44.

MRCI+Q energies; the largest difference is $0.04 \mathrm{eV}$. Both methods reproduce the experimental data rather well. For ionic-like states, the differences between the MROPT(2) and MRCI+Q excitation energies are much larger; the MROPT(2) energies correspond to experiment significantly better. The overall accuracy of the MROPT(2) method is comparable to that of MRMP(2). These two methods show the smallest deviation from the experimental excitation energies; the averaged errors for MROPT(2) and $\operatorname{MRMP}(2)$ are 0.28 and $0.23 \mathrm{eV}$ for the cc-pVDZ basis set, and 0.15 and $0.15 \mathrm{eV}$ for the cc-pVTZ basis set.

Excitation energies for eight states of benzene, ${ }^{1} E_{2 g},{ }^{1} B_{1 u}$, ${ }^{1} B_{2 u},{ }^{1} E_{1 u},{ }^{3} E_{2 g},{ }^{3} B_{1 u},{ }^{3} B_{2 u}$, and ${ }^{3} E_{1 u}$, are given in Table 2. For the ${ }^{1} E_{1 u}$ state, no solution of the MROPT(2) set of linear equations could be obtained when using iterative techniques (for details, see Sec. II.E of ref. 10). Similarly to ethylene and butadiene, the MROPT(2) and MRCI+Q excitation energies are almost identical-the largest deviation is $0.01 \mathrm{eV}$-for all covalent-like excited states of benzene: ${ }^{1} E_{2 g},{ }^{1} B_{2 u},{ }^{3} E_{2 g},{ }^{3} B_{1 u}$, and ${ }^{3} E_{1 u}$. For the other states, which are ionic-like, the differences are much larger. Again, much better correspondence to experiment is achieved for the MROPT(2) method. The overall accuracy of MROPT(2) is similar to MRMP(2); the averaged error is 0.24 and $0.19 \mathrm{eV}$, respectively.

\section{Spectroscopic Parameters of $\mathrm{N}_{2}$ and $\mathrm{O}_{2}$}

Spectroscopic parameters for six low-lying electronic states of $\mathrm{O}_{2}-b^{1} \Sigma_{g}^{+}, c{ }^{1} \Sigma_{u}^{-}, a^{1} \Delta_{g}, A^{\prime}{ }^{3} \Delta_{u}, X^{3} \Sigma_{g}^{-}$, and $A^{3} \Sigma_{u}^{+}$- are shown in Table 3. Spectroscopic parameters for eight low-lying states of $\mathrm{N}_{2}-X^{1} \Sigma_{g}^{+}, w^{1} \Delta_{u}, W^{3} \Delta_{u}, A^{3} \Sigma_{u}^{+}, a^{\prime}{ }^{1} \Sigma_{u}^{-}, a^{1} \Pi_{g}, B^{\prime}$ ${ }^{3} \Sigma_{u}^{-}$, and $B{ }^{3} \Pi_{g}-$ are shown in Table 4 . For each state, we calculate equilibrium distance $r_{e}$, harmonic vibrational frequency $\omega_{e}$, rotational constant $B_{e}$, and adiabatic and vertical excitation energies using a set of various quantum chemical methods. The results are compared to the experimental data. The last column of Tables 3 and 4 gives an average absolute deviation from experimental data for each method.
The correspondence of the MROPT(2) results to experiment is good for both molecules. For $\mathrm{O}_{2}$, the average error is only $0.013 \AA$ for $r_{e}, 21 \mathrm{~cm}^{-1}$ for $\omega_{e}, 0.02 \mathrm{~cm}^{-1}$ for $B_{e}, 0.09 \mathrm{eV}$ for adiabatic excitation energy, and $0.09 \mathrm{eV}$ for vertical excitation energy. Slightly larger are the errors obtained for $\mathrm{N}_{2}: 0.013 \AA$ for $r_{e}, 54$ $\mathrm{cm}^{-1}$ for $\omega_{e}, 0.03 \mathrm{~cm}^{-1}$ for $B_{e}, 0.26 \mathrm{eV}$ for adiabatic excitation energy, and $0.25 \mathrm{eV}$ for vertical excitation energy. In most cases, the MROPT(2) results are very similar to these of MRCI+Q. Note that the MRMP(2) method does not yield very accurate results for $\mathrm{O}_{2}$, while for $\mathrm{N}_{2}$, the accuracy of MROPT(2) and MRMP(2) is similar.

Potential energy curves for three states of $\mathrm{N}_{2}, a^{1} \Pi_{g}, A^{3} \Sigma_{u}^{+}$, and $X^{1} \Sigma_{g}^{+}$, are shown in Figure 1. Similarly, potential energy curves for three states of $\mathrm{O}_{2}, X^{3} \Sigma_{g}^{-}, A^{3} \Sigma_{g}^{+}$, and $a^{1} \Delta_{g}$, are given in Figure 2. We give these comparisons of potential energy curves calculated using various methods in order to show some characteristic behavior of the MROPT(2) method. The most interesting feature-discussed already in the preceding article $^{10}$ - is a very close resemblance of the MROPT(2) and $\mathrm{MRCI}+\mathrm{Q}$ curves. The calculated MREN(2) curves differ noticeably from the other curves. The MREN(3) and MRMP(3) curves show rather similar character, suggesting fast convergence of the perturbation series.

\section{Rotational Barrier of Ethylene}

Potential energy curves for the two lowest electronic states of ethylene, $X^{1} A_{g}$ and $1{ }^{3} B_{1 u}$, are plotted in Figure 3 as a function of torsional angle $\phi$. The computed height of the internal rotation barrier of each state is given in Table 5. All considered methodsexcept MREN(2) - predict very similar and accurate values of the rotational barrier. Again, the MROPT(2) and MRCI+Q results are very similar; the curves of these states plotted in Figure 3 almost coincide. Similarly, the curves obtained with $\operatorname{MREN}(3)$ and MRMP(3) are almost identical, even if the second-order MREN and MRMP curves are very different. This fact suggests very fast 
Table 4. Spectroscopic Parameters for Eight States of $\mathrm{N}_{2}$ Determined Using the MROPT(2) Method.

\begin{tabular}{|c|c|c|c|c|c|c|c|c|c|}
\hline \multicolumn{10}{|c|}{ Equilibrium distance $r_{e}$} \\
\hline Method & $X^{1} \Sigma_{g}^{+}$ & $w^{1} \Delta_{u}$ & $W^{3} \Delta_{u}$ & $A^{3} \Sigma_{u}^{+}$ & $a^{\prime}{ }^{1} \Sigma_{u}^{-}$ & $a^{1} \Pi_{g}$ & $B^{\prime}{ }^{3} \Sigma_{u}^{-}$ & $B{ }^{3} \Pi_{g}$ & Error \\
\hline CASSCF & 1.103 & 1.277 & 1.294 & 1.304 & 1.288 & 1.256 & 1.288 & 1.239 & 0.014 \\
\hline MRCI & 1.106 & 1.281 & 1.295 & 1.305 & 1.289 & 1.234 & 1.293 & 1.226 & 0.012 \\
\hline $\mathrm{MRCI}+\mathrm{Q}$ & 1.107 & 1.285 & 1.298 & 1.307 & 1.292 & 1.233 & 1.297 & 1.228 & 0.013 \\
\hline MREN(2) & 1.106 & 1.275 & 1.288 & 1.295 & 1.281 & 1.201 & 1.288 & 1.195 & 0.010 \\
\hline MREN(3) & 1.107 & 1.286 & 1.300 & 1.309 & 1.295 & 1.256 & 1.299 & 1.248 & 0.020 \\
\hline MRMP(2) & 1.109 & 1.286 & 1.299 & 1.308 & 1.292 & 1.222 & 1.298 & 1.213 & 0.011 \\
\hline MRMP(3) & 1.107 & 1.281 & 1.295 & 1.307 & 1.289 & 1.231 & 1.291 & 1.222 & 0.011 \\
\hline MROPT(2) & 1.107 & 1.286 & 1.298 & 1.307 & 1.293 & 1.229 & 1.298 & 1.228 & 0.013 \\
\hline Exp. $^{\text {a }}$ & 1.098 & 1.268 & - & 1.287 & 1.276 & 1.220 & 1.278 & 1.213 & \\
\hline
\end{tabular}

Harmonic vibrational frequency $\omega_{e}$

\begin{tabular}{|c|c|c|c|c|c|c|c|c|c|}
\hline Method & $X^{1} \Sigma_{g}^{+}$ & $w^{1} \Delta_{u}$ & $W^{3} \Delta_{u}$ & $A^{3} \Sigma_{u}^{+}$ & $a^{\prime}{ }^{1} \Sigma_{u}^{-}$ & $a{ }^{1} \Pi_{g}$ & $B^{\prime}{ }^{3} \Sigma_{u}^{-}$ & $B{ }^{3} \Pi_{g}$ & Error \\
\hline CASSCF & 2404 & 1555 & 1475 & 1412 & 1510 & 1473 & 1504 & 1560 & 68 \\
\hline MRCI & 2356 & 1503 & 1444 & 1383 & 1476 & 1671 & 1449 & 1691 & 47 \\
\hline $\mathrm{MRCI}+\mathrm{Q}$ & 2335 & 1487 & 1425 & 1365 & 1432 & 1716 & 1431 & 1767 & 63 \\
\hline $\operatorname{MREN}(2)$ & 2381 & 1559 & 1488 & 1443 & 1498 & 1828 & 1501 & 1902 & 50 \\
\hline MREN(3) & 2359 & 1486 & 1423 & 1363 & 1471 & 1470 & 1419 & 1488 & 109 \\
\hline $\operatorname{MRMP(2)}$ & 2362 & 1521 & 1452 & 1385 & 1502 & 1601 & 1472 & 1668 & 49 \\
\hline MRMP(3) & 2369 & 1520 & 1455 & 1394 & 1491 & 1645 & 1468 & 1688 & 43 \\
\hline MROPT(2) & 2356 & 1495 & 1439 & 1377 & 1482 & 1649 & 1439 & 1678 & 54 \\
\hline Exp. $^{a}$ & 2359 & 1559 & 1501 & 1461 & 1530 & 1694 & 1517 & 1733 & \\
\hline
\end{tabular}

Rotational constant $B_{e}$

\begin{tabular}{|c|c|c|c|c|c|c|c|c|c|}
\hline Method & $X^{1} \Sigma_{g}^{+}$ & $w^{1} \Delta_{u}$ & $W^{3} \Delta_{u}$ & $A^{3} \Sigma_{u}^{+}$ & $a^{\prime}{ }^{1} \Sigma_{u}^{-}$ & $a^{1} \Pi_{g}$ & $B^{\prime}{ }^{3} \Sigma_{u}^{-}$ & $B{ }^{3} \Pi_{g}$ & Error \\
\hline CASSCF & 1.98 & 1.48 & 1.44 & 1.41 & 1.45 & 1.53 & 1.45 & 1.57 & 0.03 \\
\hline MRCI & 1.97 & 1.47 & 1.43 & 1.41 & 1.45 & 1.58 & 1.44 & 1.60 & 0.03 \\
\hline $\mathrm{MRCI}+\mathrm{Q}$ & 1.96 & 1.46 & 1.43 & 1.41 & 1.44 & 1.58 & 1.43 & 1.60 & 0.03 \\
\hline $\operatorname{MREN}(2)$ & 1.97 & 1.48 & 1.45 & 1.44 & 1.47 & 1.67 & 1.45 & 1.69 & 0.02 \\
\hline MREN(3) & 1.96 & 1.46 & 1.42 & 1.40 & 1.44 & 1.53 & 1.43 & 1.54 & 0.05 \\
\hline $\operatorname{MRMP}(2)$ & 1.96 & 1.45 & 1.43 & 1.41 & 1.44 & 1.61 & 1.43 & 1.63 & 0.02 \\
\hline $\operatorname{MRMP}(3)$ & 1.96 & 1.47 & 1.44 & 1.41 & 1.45 & 1.59 & 1.44 & 1.61 & 0.03 \\
\hline MROPT(2) & 1.96 & 1.46 & 1.43 & 1.41 & 1.44 & 1.59 & 1.43 & 1.60 & 0.03 \\
\hline Exp. $^{a}$ & 2.00 & 1.50 & - & 1.45 & 1.48 & 1.62 & 1.47 & 1.64 & \\
\hline
\end{tabular}

Adiabatic excitation energy

\begin{tabular}{|c|c|c|c|c|c|c|c|c|c|}
\hline Method & $X^{1} \Sigma_{g}^{+}$ & $w^{1} \Delta_{u}$ & $W^{3} \Delta_{u}$ & $A^{3} \Sigma_{u}^{+}$ & $a^{\prime}{ }^{1} \Sigma_{u}^{-}$ & $a^{1} \Pi_{g}$ & $B^{\prime}{ }^{3} \Sigma_{u}^{-}$ & $B{ }^{3} \Pi_{g}$ & Error \\
\hline CASSCF & 0.00 & 10.16 & 7.98 & 6.39 & 9.31 & 11.01 & 9.27 & 9.44 & 1.19 \\
\hline MRCI & 0.00 & 9.11 & 7.39 & 6.02 & 8.56 & 8.88 & 8.29 & 7.55 & 0.14 \\
\hline $\mathrm{MRCI}+\mathrm{Q}$ & 0.00 & 8.84 & 7.26 & 5.92 & 8.41 & 8.10 & 8.02 & 6.99 & 0.24 \\
\hline $\operatorname{MREN}(2)$ & 0.00 & 8.84 & 7.05 & 5.86 & 8.14 & 7.73 & 7.84 & 6.12 & 0.52 \\
\hline MREN(3) & 0.00 & 9.07 & 7.34 & 5.99 & 8.54 & 9.54 & 8.30 & 8.24 & 0.34 \\
\hline $\operatorname{MRMP}(2)$ & 0.00 & 8.63 & 6.89 & 5.55 & 8.04 & 8.13 & 7.85 & 6.69 & 0.49 \\
\hline MRMP(3) & 0.00 & 9.02 & 7.33 & 6.07 & 8.49 & 9.29 & 8.35 & 7.81 & 0.23 \\
\hline MROPT(2) & 0.00 & 8.79 & 7.28 & 5.96 & 8.42 & 7.92 & 7.99 & 7.02 & 0.26 \\
\hline Exp. $^{a}$ & 0.00 & 8.94 & 7.42 & 6.22 & 8.45 & 8.59 & 8.22 & 7.39 & \\
\hline
\end{tabular}


Table 4. (Continued)

\begin{tabular}{|c|c|c|c|c|c|c|c|c|c|}
\hline \multicolumn{10}{|c|}{ Vertical excitation energy } \\
\hline Method & $X^{1} \Sigma_{g}^{+}$ & $w^{1} \Delta_{u}$ & $W^{3} \Delta_{u}$ & $A^{3} \Sigma_{u}^{+}$ & $a^{\prime}{ }^{1} \Sigma_{u}^{-}$ & $a^{1} \Pi_{g}$ & $B^{\prime}{ }^{3} \Sigma_{u}^{-}$ & $B{ }^{3} \Pi_{g}$ & Error \\
\hline CASSCF & 0.00 & 11.65 & 9.66 & 8.19 & 10.94 & 12.07 & 10.90 & 10.34 & 1.41 \\
\hline MRCI & 0.00 & 10.63 & 9.06 & 7.78 & 10.18 & 9.76 & 9.95 & 8.34 & 0.26 \\
\hline $\mathrm{MRCI}+\mathrm{Q}$ & 0.00 & 10.40 & 8.96 & 7.70 & 10.05 & 8.99 & 9.72 & 7.83 & 0.13 \\
\hline $\operatorname{MREN}(2)$ & 0.00 & 10.29 & 8.64 & 7.52 & 9.68 & 8.30 & 9.44 & 6.65 & 0.48 \\
\hline MREN(3) & 0.00 & 10.61 & 9.04 & 7.78 & 10.20 & 10.57 & 10.00 & 9.19 & 0.50 \\
\hline $\operatorname{MRMP}(2)$ & 0.00 & 10.19 & 8.59 & 7.33 & 9.69 & 8.89 & 9.55 & 7.38 & 0.31 \\
\hline $\operatorname{MRMP}(3)$ & 0.00 & 10.52 & 8.99 & 7.87 & 10.09 & 10.10 & 9.98 & 8.54 & 0.32 \\
\hline MROPT(2) & 0.00 & 10.34 & 8.98 & 7.74 & 10.06 & 8.70 & 8.99 & 7.84 & 0.25 \\
\hline Exp. $^{\text {b }}$ & 0.00 & 10.27 & 8.88 & 7.75 & 9.92 & 9.31 & 9.67 & 8.04 & \\
\hline
\end{tabular}

Results obtained using other methods are given for comparison. Distances are given in $\AA$, vibrational frequencies and rotational constants in $\mathrm{cm}^{-1}$, and excitation energies in $\mathrm{eV}$. Error is computed as an average absolute deviation from the experimental results. For $W^{3} \Delta_{u}$, for which no experimental values of $r_{e}$ and $B_{e}$ are available, the corresponding $\mathrm{MRCI}+\mathrm{Q}$ values are used instead.

${ }^{\mathrm{a}}$ Ref. 45 .

${ }^{\mathrm{b}}$ Ref. 46.

convergence of the energy perturbation series for ethylene. Somewhat larger deviation from experiment for the barriers calculated with MREN(2) may be associated with large size-consistency deviations for this method. Note that for the MREN(3) - for which size-inconsistency is less severe-the correspondence of calculated barriers to experiment is already very good.

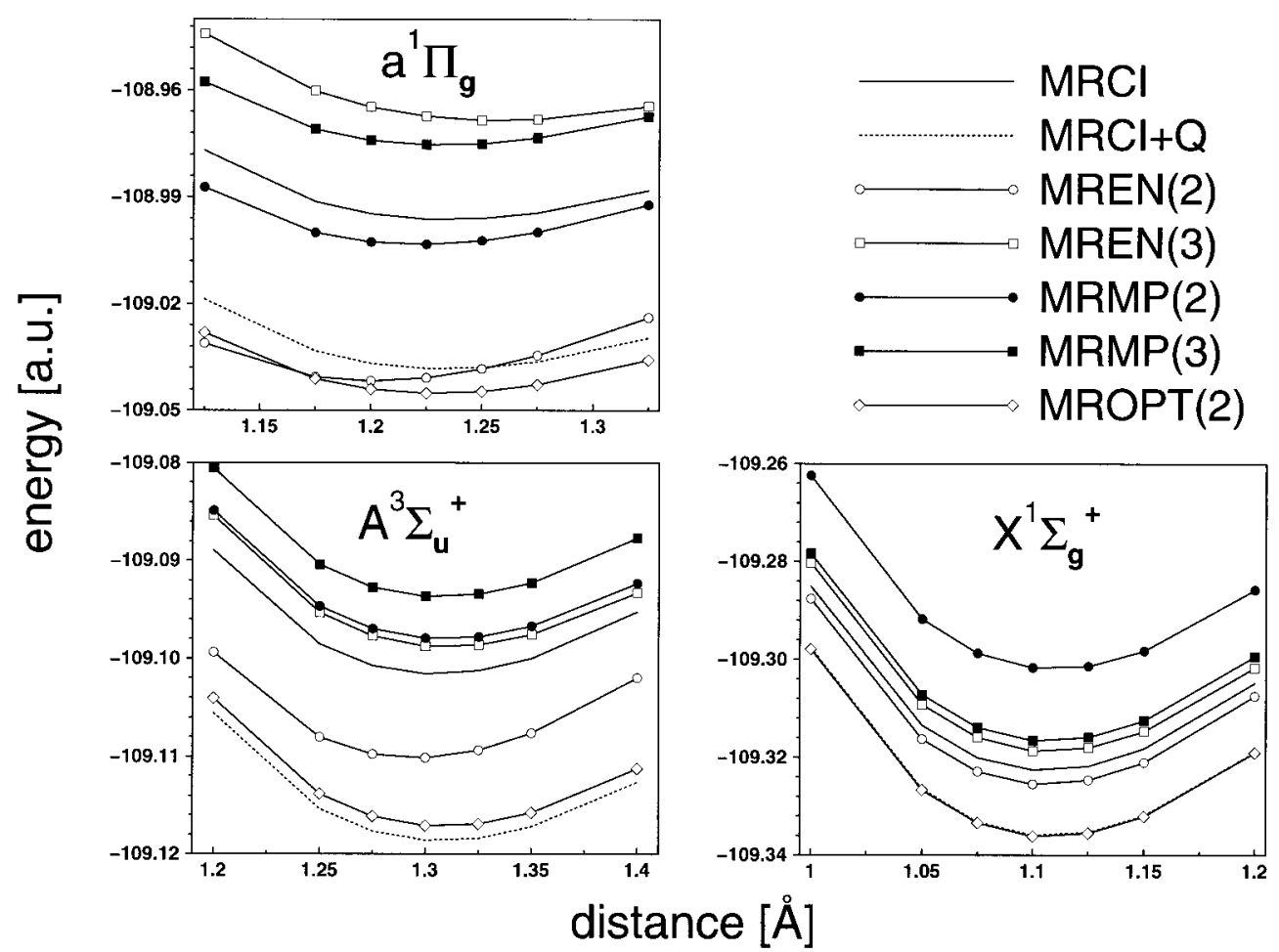

Figure 1. Comparison of potential energy curves in the equilibrium region for three electronic states of $\mathrm{N}_{2}, a{ }^{1} \Pi_{g}, A{ }^{3} \Sigma_{u}^{+}$, and $X^{1} \Sigma_{g}^{+}$, calculated using various methods. Note that the MROPT(2) and $\mathrm{MRCI}+\mathrm{Q}$ curves coincide for the $X^{1} \Sigma_{g}^{+}$state. 


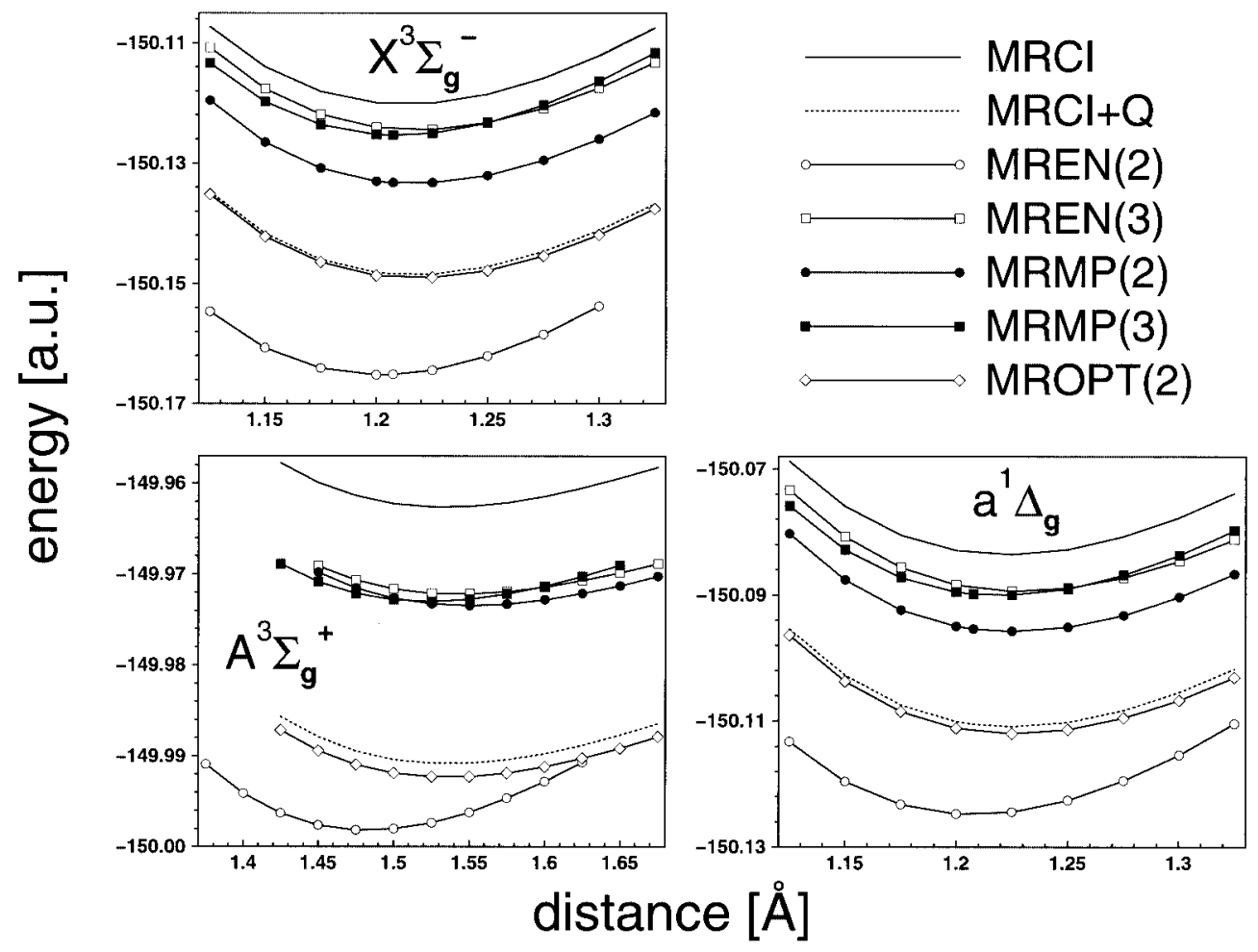

Figure 2. Comparison of potential energy curves in the equilibrium region for three electronic states of $\mathrm{O}_{2}, X^{3} \Sigma_{g}^{-}, A^{3} \Sigma_{g}^{+}$, and $a^{1} \Delta_{g}$, calculated using various methods.

\section{Conclusions}

MROPT(2) has been applied to calculations on some molecular properties of the ground and excited states of a set of five molecules. We have calculated: vertical excitation energies of two states of ethylene, four of butadiene, and eight of benzene, spectroscopic constants-including equilibrium distances, harmonic vibrational frequencies, rotational constants, and vertical and adiabatic excitation energies-for six low-lying states of $\mathrm{O}_{2}$ and eight of $\mathrm{N}_{2}$, and potential energy curve of ethylene as a function of the torsional angle $\phi$. The calculated results are compared with those obtained with second- and third-order multireference PT using Møller-Plesset and Epstein-Nesbet partitionings. We give also the results from computations using MRCI and Davidson-corrected MRCI.

The parameters $\Delta_{i}$, obtained by solving eq. (5), can have a very wide range of magnitudes: the largest can exceed a value of a few thousands of hartree, being either positive or negative. It is very difficult to supply more information on their values, owing to their abundance. The only applicable approach that can give some insight is the statistical one. The parameters $\Delta_{i}$ have an approximately Gaussian-like distribution with maximum being around 0 hartree and $90 \%$ of them located in the range between -5 and 5 hartree. The position of the maximum tends to be shifted somewhat to negative values for large FOCI spaces. Similarly, the distribution curve tends to be narrower for small dimensions of FOCI.
On the whole, MROPT(2) has shown very good performance. The deviations from available experimental data are rather small: $\approx 0.2 \mathrm{eV}$ for excitation energy, $0.01 \AA$ for bond length, and $\approx 50$ $\mathrm{cm}^{-1}$ for harmonic vibrational frequency. The computational cost of the MROPT(2) method-approximately similar to the cost of MRCI-is rather high when compared to other second-order perturbative treatments; the bottleneck of MROPT(2) is a necessity of solving a large set of linear equations. Some interesting features have been found in the performance of MRPTs and MRCI:

1. The MROPT(2) results on excitation energies, spectroscopic constants, and potential energy curves are very similar to those of $\mathrm{MRCI}+\mathrm{Q}$.

2. The second-order MRPT results are better than the third-order ones when using the Møller-Plesset partitioning.

3. Properties computed by the third-order Epstein-Nesbet and Møller-Plesset PTs, MREN(3), and MRMP(3) are also very similar, probably suggesting fast convergence of the perturbational series.

4. At the second-order, MREN usually gives poorer results than MROPT and MRMP.

Some comments on the first two points are given below.

MROPT(2) and MRCI+Q usually give very similar results; an exception is a noticeably larger difference between MROPT(2) and $\mathrm{MRCI}+\mathrm{Q}$ for valence excitation energies for ionic-like states of ethylene, butadiene, and benzene. The largest deviation is 0.04 


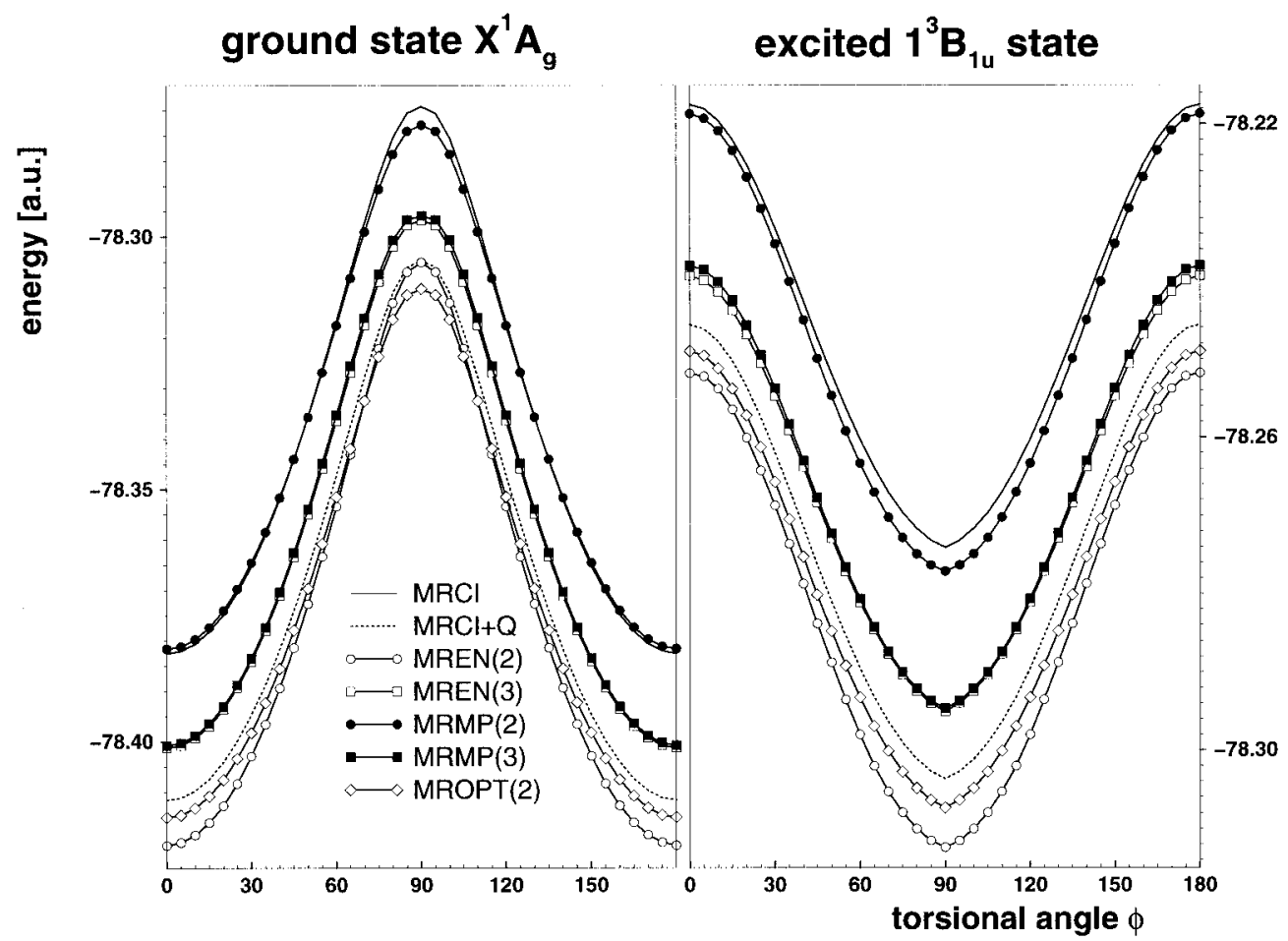

Figure 3. Potential energy curves for two lowest electronic states of ethylene, $X{ }^{1} A_{g}$ and $1{ }^{3} B_{1 u}$, as a function of torsional angle $\phi$.

$\mathrm{eV}$ for covalent-like states while it is $0.53 \mathrm{eV}$ for ionic-like states. This large difference in ionic states is probably due to the choice of active spaces. The active spaces used in the present article are minimal ones that take into account the $\pi$ correlation. As suggested from the basis set effect in the section Rotational Barrier of Ethylene, polarization effect is more important for the ionic-like states. Larger active spaces including higher polarization effect

Table 5. Barrier of Internal Rotation (kcal/mol) for Two States of Ethylene Calculated Using the MROPT(2) Method.

\begin{tabular}{lcc}
\hline Method & $X{ }^{1} A_{g}$ & $1{ }^{3} B_{1 u}$ \\
\hline CASSCF & 67.4 & 33.8 \\
MRCI & 67.9 & 35.5 \\
MRCI+Q & 67.0 & 36.4 \\
MREN(2) & 72.4 & 38.0 \\
MREN(3) & 65.5 & 34.9 \\
MRMP(2) & 65.0 & 36.7 \\
MRMP(3) & 65.8 & 35.5 \\
MROPT(2) & 65.6 & 36.6 \\
Exp. & $\approx 65^{\mathrm{a}}$ & $\approx 35^{\mathrm{b}}$ \\
\hline
\end{tabular}

Results obtained using other methods are given for comparison.

${ }^{\mathrm{a}}$ Refs. 47, 48.

${ }^{b}$ Estimated from the difference between the vertical excitation energy of 1 ${ }^{3} B_{1 u}$ (Refs. 47, 48) and barrier height for the ground state; we assume that the lowest triplet and singlet states are degenerated for the torsional angle $\phi=90^{\circ}$. will improve the description of the ionic-like states and reduce the difference between MROPT(2) and MRCI+Q.

A better performance of the second-order MRMP than the third-order MRMP, mentioned above, may look strange. However, this irregularity can also be seen in single reference MP theory and it is not a particular feature of multireference MP theory. See, for example, systematic studies on the convergence of MP series given in refs. 29-31. These articles also present some other unexpected features of the MP theory, for example, oscillatory behavior of the MP series in high orders. It would be interesting to pursue the origin of the strange behavior also in multireference MP PT. However, we do not discuss it here any longer, because it is not a subject of the present article.

Now we can safely say that the new method using the optimized partitioning, MROPT(2), gives accurate results comparable to MRMP(2) and MRCI+Q on molecular properties, and, moreover, it can well reproduce experimental values.

\section{Acknowledgments}

The present research was supported in part by a Grant-in-Aid for Specially Promoted Research, "Simulations and Dynamics of Real Molecular Systems," from the Ministry of Education, Culture, Sports, Science, and Technology of Japan. H.W. would like to thank Prof. Hans Lischka for the assistance with the COLUMBUS $a b$ initio electronic structure program, and Dr. Maciej Bobrowski for help with geometry optimization of ethylene. All the presented 
calculations were performed with the COLUMBUS MRCI code,$^{20-23}$ modified for needs of perturbation theory.

\section{References}

1. Møller, C.; Plesset, M. S. Phys Rev 1934, 46, 618.

2. Epstein, P. S. Phys Rev 1926, 28, 695.

3. Nesbet, R. K. Proc R Soc London 1955, A230, 312.

4. Amos, A. T. J Chem Phys 1970, 52, 603.

5. Szabados, Á.; Surján, P. R. Chem Phys Lett 1999, 308, 303.

6. Surján, P. R.; Szabados, Á. J Chem Phys 2000, 112, 4438.

7. Finley, J. P. J Chem Phys 2000, 112, 6997.

8. Finley, J. P.; Chaudhuri, R. K.; Freed, K. F. J Chem Phys 1995, 103, 4990.

9. Finley, J. P.; Chaudhuri, R. K.; Freed, K. F. Phys Rev A 1996, 54, 343.

10. Witek, H. A.; Nakano, H.; Hirao, K. J Chem Phys 2003, 118, 8197.

11. Laidig, W. D.; Bartlett, R. J. Chem Phys Lett 1984, 104, 424.

12. Laidig, W. D.; Saxe, P.; Bartlett, R. J. J Chem Phys 1987, 86, 887.

13. Surján, P. R.; Szabados, Á.; Szekeres, Z. Int J Quantum Chem 2002, 90, 1309.

14. Dyson, F. J. Phys Rev 1949, 75, 1736.

15. Herzberg, G. Electronic Spectra of Polyatomic Molecules; Van Nostrand: New York, 1966; p 629.

16. Haugen, W.; Traetteberg, M. Acta Chem Scand 1966, 20, 1726.

17. Dunning Jr., T. H. J Chem Phys 1989, 90, 1007.

18. Pierloot, K.; Dumez, B.; Widmark, P.-O.; Roos, B. O. Theor Chim Acta 1995, 90, 87.

19. Widmark, P.-O.; Malmqvist, P. Å.; Roos, B. O. Theor Chim Acta 1990, 77, 291.

20. Lischka, H.; Shepard, R.; Brown, F. B.; Shavitt, I. Int J Quantum Chem 1981, S15, 91.

21. Shepard, R.; Shavitt, I.; Pitzer, R. M.; Comeau, D. C.; Pepper, M.; Lischka, H.; Szalay, P.; Ahlrichs, R.; Brown, F. B.; Zhao, J. G. Int J Quantum Chem 1988, S22, 149.

22. Lischka, H.; Shepard, R.; Pitzer, R. M.; Shavitt, I.; Dallos, M.; Müller, T.; Szalay, P. G.; Seth, M.; Kedziora, G. S.; Yabushita, S.; Zhang, Z. Y. Phys Chem Chem Phys 2001, 3, 664.

23. Lischka, H.; Shepard, R.; Shavitt, I.; Pitzer, R. M.; Dallos, M.; Müller, Th.; Szalay, P. G.; Brown, F. B.; Ahlrichs, R.; Böhm, H. J.; Chang, A.;
Comeau, D. C.; Gdanitz, R.; Dachsel, H.; Ehrhardt, C.; Ernzerhof, M.; Höchtl, P.; Irle, S.; Kedziora, G. S.; Kovar, T.; Parasuk, V.; Pepper, M. J. M.; Scharf, P.; Schiffer, H.; Schindler, M.; Schüler, M.; Seth, M.; Stahlberg, E. A.; Zhao, J. G.; Yabushita, S.; Zhang, Z. COLUMBUS, release 5.8; an ab initio electronic structure program; 2001.

24. Siegbahn, P. E. M. Chem Phys Lett 1978, 55, 386.

25. Hirao, K.; Nakano, H.; Nakayama, K. J Chem Phys 1996, 105, 9227.

26. Nakayama, K.; Nakano, H.; Hirao, K. Int J Quantum Chem 1998, 66, 157.

27. Hashimoto, T.; Nakano, H.; Hirao, K. J Chem Phys 1996, 104, 6244.

28. Hashimoto, T.; Nakano, H.; Hirao, K. J Mol Struct (Theochem) 1998, $451,25$.

29. Cremer, D.; He, Z. J Chem Phys 1996, 100, 6173.

30. Dunning Jr., T. H.; Peterson, K. A. J Chem Phys 1998, 108, 4761.

31. Olsen, J.; Christiansen, O. V.; Koch, H.; Jørgensen, P. J Chem Phys 1996, 105, 5082.

32. Petrongolo, C.; Buenker, R. J.; Peyerimhoff, S. D. J Chem Phys 1982, 76,3655 .

33. McMurchie, L. E.; Davidson, E. R. J Chem Phys 1977, 67, 5613.

34. Lindh, R.; Roos, B. O. Int J Quantum Chem 1989, 35, 813.

35. Serrano-Andrés, L.; Merchán, M.; Nebot-Gil, I.; Lindh, R.; Roos, B. O. J Chem Phys 1993, 98, 3151.

36. van Veen, E. H. Chem Phys Lett 1976, 41, 540.

37. Flicker, W. H.; Mosher, O. A.; Kuppermann, A. Chem Phys Lett 1975, $36,56$.

38. McDiarmid, R. Chem Phys Lett 1992, 188, 423.

39. Nakashima, N.; Inoue, H.; Sumitani, M.; Yoshihara, K. J Chem Phys 1980, 73, 5976.

40. Hiraya, A.; Shobatake, K. J Chem Phys 1991, 94, 7700.

41. Astier, R.; Mayer, Y. H. Chem Phys Lett 1969, 3, 399.

42. Doering, J. P. J Chem Phys 1969, 51, 2866.

43. Krupenie, P. H. J Phys Chem Ref Data 1972, 1, 456.

44. Slanger, T. G.; Cosby, P. C. J Phys Chem 1988, 92, 267.

45. Huber, K. P.; Herzberg, G. Constants of Diatomic Molecules; Van Nostrand: New York, 1979; pp 418-420.

46. Oddershede, J.; Grüner, N. E.; Diercksen, G. H. F. Chem Phys 1985, 97, 303.

47. Douglas, J. E.; Rabinovitch, B. S.; Looney, F. S. J Chem Phys 1955, 23, 315.

48. Merer, A. J.; Mulliken, R. S. Chem Rev 1969, 69, 639. 